\title{
CORRIGENDUM
}

\section{Childhood obesity: evidence for distinct early and late environmental determinants a 12-year longitudinal cohort study (EarlyBird 62)}

M Mostazir, A Jeffery, L Voss and T Wilkin

International Journal of Obesity (2016) 40, 380; doi:10.1038/ijo.2015.248

Correction to: International Journal of Obesity (2015) 39, 1057-1062; doi:10.1038/ijo.2015.68

Since the publication of this article the authors have noticed an error in the affiliations.

Affiliation for Mohammod Mostazir should be 'College of Life and Environmental Sciences (CLES), University of Exeter, Exeter, UK'.

Hence, affiliations should read as follows:

M Mostazir ${ }^{1}$, A Jeffery ${ }^{2}$, L Voss ${ }^{2}$ and T Wilkin ${ }^{3}$
${ }^{1}$ College of Life and Environmental Sciences (CLES), Wellcome Trust Biomedical Informatics Hub, University of Exeter, Exeter, UK

${ }^{2}$ Department of Endocrinology and Metabolism, Plymouth University Peninsula School of Medicine and Dentistry, Plymouth, UK

${ }^{3} \mathrm{HHR}$, University of Exeter Medical School, Exeter, UK

The corrected article html and online pdf versions have also been amended. The authors would like to apologize for the error. 